\title{
ARTICLE
}

Received 25 Nov 2013 | Accepted 10 Sep 2014 | Published 30 Oct $2014 \quad$ DOl: 10.1038/ncomms6229

\section{Three-dimensional analysis of Nafion layers in fuel cell electrodes}

\author{
M. Lopez-Haro ${ }^{1,2}$, L. Guétaz'1,3, T. Printemps ${ }^{1,4}$, A. Morin 1,3, S. Escribano1,3, P.-H. Jouneau1,2, \\ P. Bayle-Guillemaud ${ }^{1,2}$, F. Chandezon ${ }^{1,5,6} \&$ G. Gebel ${ }^{1,3,5,6}$
}

Proton exchange membrane fuel cell is one of the most promising zero-emission power sources for automotive or stationary applications. However, their cost and lifetime remain the two major key issues for a widespread commercialization. Consequently, much attention has been devoted to optimizing the membrane/electrode assembly that constitute the fuel cell core. The electrodes consist of carbon black supporting Pt nanoparticles and Nafion as the ionomer binder. Although the ionomer plays a crucial role as ionic conductor through the electrode, little is known about its distribution inside the electrode. Here we report the three-dimensional morphology of the Nafion thin layer surrounding the carbon particles, which is imaged using electron tomography. The analyses reveal that doubling the amount of Nafion in the electrode leads to a twofold increase in its degree of coverage of the carbon, while the thickness of the layer, around $7 \mathrm{~nm}$, is unchanged.

\footnotetext{
${ }^{1}$ University Grenoble Alpes, Grenoble F-38000, France. ${ }^{2}$ CEA, INAC-SP2M, LEMMA, Grenoble F-380054, France. ${ }^{3}$ CEA, LITEN, Grenoble F-38054, France. ${ }^{4}$ CEA, LETI, Minatec Campus, Grenoble F-38054, France. ${ }^{5}$ CNRS, SPrAM, Grenoble F-38054, France. ${ }^{6}$ CEA, INAC-SPrAM, Grenoble F-38054, France. Correspondence and requests for materials should be addressed to F.C. (email: frederic.chandezon@cea.fr) or to G.G. (email: gerard.gebel@cea.fr).
} 
$\mathrm{T}$ he development of alternative energies is a critical issue because of the increasing scarcity of fossil fuels and the pollution problems associated with their use $\mathrm{e}^{1,2}$. In this regard, proton exchange membrane fuel cells (PEMFCs) are recognized as one of the most promising energy-converting devices to obtain electrical energy through electrochemical reactions without generation of pollutants ${ }^{3,4}$. The membrane electrode assembly (MEA) is the key component influencing the performance, the long-term stability and the production costs of a PEMFC. It is composed of a proton-conducting membrane coated on each side with electrodes involving a catalyst layer where the electrochemical reactions take place. The catalyst layer itself is a composite porous nanomaterial generally consisting of carbon black (CB) supporting platinum nanoparticles (the catalyst) and using an ionomer as both binder and electrolyte ${ }^{4-6}$. Reference materials for the proton exchange membrane and for the ionomer in the catalyst layer are perfluorosulfonated ionomers, whose most famous example is commercialized under the trade name Nafion. In the electrodes, the electrochemical performance can be limited by the transport of protons, electrons or reactant gases $\left(\mathrm{H}_{2}\right.$ and $\left.\mathrm{O}_{2}\right)$ to the catalyst sites. Consequently, the MEA multiscale microstructure strongly influences the performance of a $\mathrm{PEMFC}^{7-9}$.

Much research work has been devoted to enhance the microstructure in the cathode electrode in order to optimize the degree of Pt utilization under operation that is estimated to be of $\sim 10-20 \%$ in state-of-the-art electrodes ${ }^{10}$. This optimization is necessary to reduce the Pt loading and therefore the cost of a PEMFC. The structure of the ionomer network inside the active layer is probably the main factor that affects the degree of $\mathrm{Pt}$ utilization. Indeed, this must ensure both ionic contact with a maximum of $\mathrm{Pt}$ nanoparticles and the connectivity of the ionic conduction paths to the membrane without inhibiting the gas diffusivity. Despite the crucial role of the ionomer layer, there exist presently very few experimental data on the ionomer distribution within the electrode. Hitchcock and co-workers ${ }^{11}$ demonstrated the capabilities of synchrotron soft X-ray microscopy tomography method for mapping in $3 \mathrm{D}$ the ionomer across the electrode; however, at a more local length scale, the currently only available information about the morphology of the Nafion layer surrounding the $\mathrm{CB}$ is the thickness, which was estimated to be $\sim 5-10 \mathrm{~nm}$ from selected transmission electron microscopy (TEM) images ${ }^{12,13}$. The present work shows for the first time a 3D characterization of this Nafion layer at the nanometre scale. 3D reconstructions obtained by electron tomography can indeed provide critical parameters for understanding and improving such structures, for example, the thickness and the degree of coverage of the $\mathrm{CB}$ particles by the ionomer.

\section{Results}

Electron tomography. Electron tomography performed in a highangle annular dark-field scanning transmission electron microscope (HAADF-STEM) is a powerful method to characterize nanomaterials in $3 \mathrm{D}$ at the nanometre scale from a series of $2 \mathrm{D}$ electron microscopy images ${ }^{14,15}$. Indeed, the contrast in HAADFSTEM images depends monotonically on the sample thickness, which is consistent with tomography criteria for the reconstruction and shows some chemical sensitivity ${ }^{16}$. This makes it ideal to study materials with very different average atomic numbers. Thereby, electron tomography is quite widely used to study the nanoparticle distribution on their support in different catalyst materials ${ }^{17}$ and particularly in fuel cell electrodes ${ }^{18-21}$.

In the case of MEAs, the Nafion ionomer consists of a polytetrafluoroethylene (Teflon) backbone with perfluorovinyl ether chains terminated by sulfonate groups as side chains. This ionomer does not present sufficient electronic contrast as compared with the $\mathrm{CB}$ to be properly imaged in a HAADFSTEM mode. However, the electron density contrast can be dramatically enhanced by selectively staining the ionic domains with $\mathrm{Cs}^{+}$ions $^{22}$. For this purpose, we studied a model active layer that is prepared with an ink consisting of $\mathrm{CB}$ instead of $\mathrm{CB}$ supporting $\mathrm{Pt}$ nanoparticles in order to avoid perturbing the STEM images with the high-contrast $\mathrm{Pt}$ nanoparticles ${ }^{21}$. The ionomer was stained with $\mathrm{Cs}^{+}$ions after coating the ink on the gas diffusion layer using the usual method as for processing an electrode. Two samples were prepared from inks with different compositions: ink for sample 1 has $33 \mathrm{wt} \%$ content of Nafion (Nafion $/ \mathrm{CB}=0.5 \mathrm{w} / \mathrm{w}$ ), which is the widely used content in the electrode of PEMFCs, whereas sample 2 was prepared from an ink containing nearly twice as less Nafion, that is, $17 \mathrm{wt} \%$ $($ Nafion $/ \mathrm{CB}=0.2 \mathrm{w} / \mathrm{w})$

Figure 1 shows a representative HAADF-STEM image of $\mathrm{Cs}^{+}$. stained sample 1 (Fig. 1a) and sample 2 (Fig. 1b) extracted from a tomography series taken at different tilt angles. Bright areas surrounding darker agglomerated regions are clearly observed in
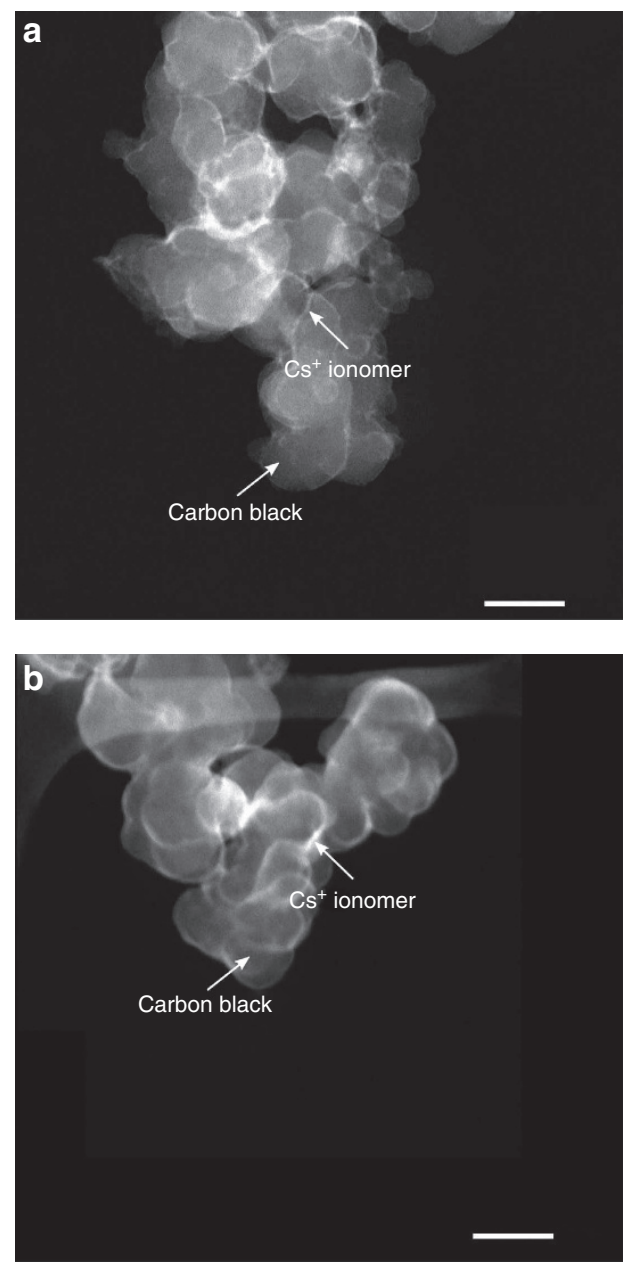

Figure 1 | 2D imaging of the Nafion/CB nanocomposites in the electrodes of PEMFCs. (a) HAADF-STEM representative micrograph of sample 1 with composition Nafion/CB $=0.5 \mathrm{w} / \mathrm{w}$. As the intensity of the HAADF-STEM signal is roughly proportional to $Z^{2}$, the highest intensities thus correspond to the presence of the $\mathrm{Cs}^{+}$ionomer $\left(Z_{\mathrm{Cs}}=55\right)$, whereas the darker regions are attributed to the $C B$ support $\left(Z_{C B}=6\right)$. (b) Same for sample 2 with composition Nafion $/ C B=0.2 \mathrm{w} / \mathrm{w}$. The scale bars correspond to $100 \mathrm{~nm}$. 
a
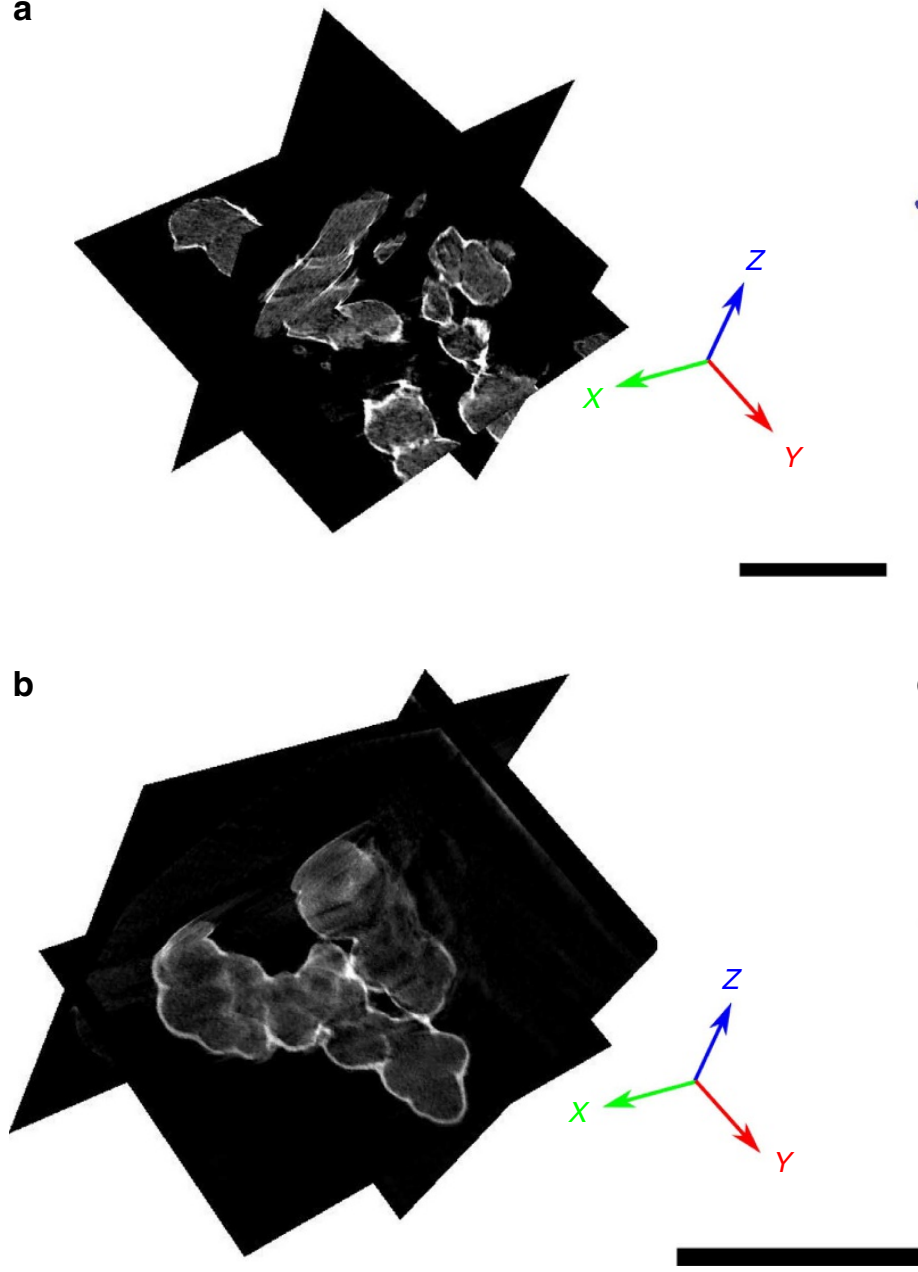

C

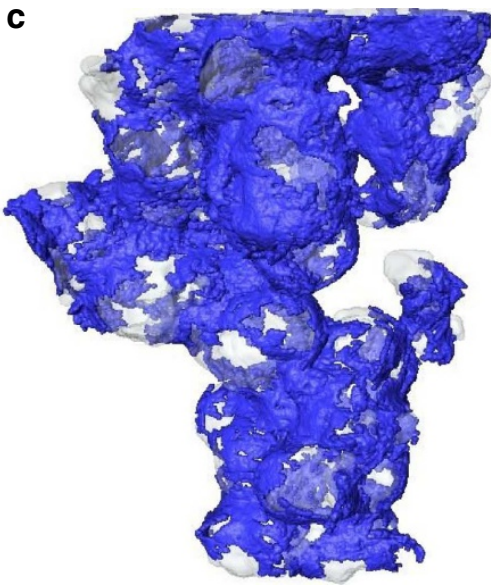

d

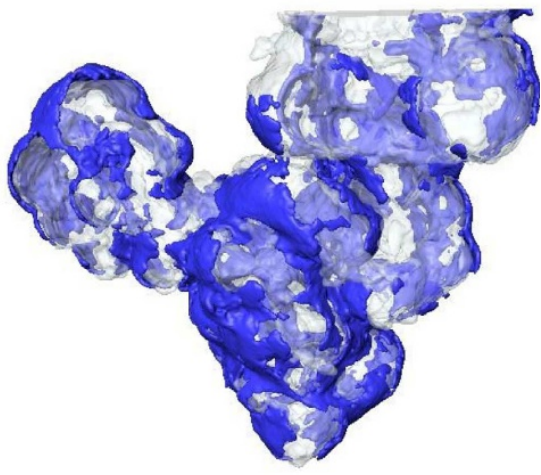

Figure 2 | Electron tomography imaging of the Nafion layer deposited on CB in the electrodes of PEMFCs. After reconstructing the volume, orthoslices corresponding to one slice of the tomogram can be extracted in planes of whatever orientation as compared with the direction. (a,b) Three representative orthoslices parallel or perpendicular to the incident electron beam axis (along the $z$ direction) extracted from the reconstructed tomograms for sample 1 (Nafion/CB $=0.5 \mathrm{w} / \mathrm{w}$ ) and sample $2($ Nafion $/ C B=0.2 \mathrm{w} / \mathrm{w}$ ), respectively. (c) 3D-rendered volume of sample 1 obtained by segmentation of the HAADFSTEM-reconstructed tomogram. The blue and grey regions correspond to the $\mathrm{Cs}^{+}$-stained ionomer and to the CB support, respectively. (d) Same for sample 2. The scale bars correspond to $200 \mathrm{~nm}$.

the micrographs that correspond to the Nafion layer. Such images can provide an estimate of the thickness of the ionomer layer but not a full picture of its distribution because of the 3D structure of the sample, thus not providing any information regarding the degree of coverage of the $\mathrm{CB}$ by the ionomer. The present work shows that the combination of HAADF-STEM tomography with the most advanced tomographic reconstruction algorithm can (i) provide a full $3 \mathrm{D}$ view of such structure and (ii) by tomogram analysis allows to extract relevant quantitative information.

To reconstruct the 3D structure, we used an algorithm based on compressed sensing ${ }^{23}$. The main advantage of this algorithm is to minimize artefacts such as the false elongation resulting from the 'missing wedge', thus providing a higher accuracy of reconstruction even with a low number of projections (see Supplementary Methods and Supplementary Fig. 1 for more details). Orthoslices for samples 1 and 2 are displayed in Fig. 2a,b, respectively. As one can notice, the Nafion layer can be clearly observed on the supporting CB particles in the orthoslices.

A carefully controlled binarization process was performed to separate the ionomer voxels from the $\mathrm{CB}$ ones in order to segment the tomograms and extract the most relevant morphological parameters of the Nafion layer (Fig. 3 and Supplementary
Fig. 2). The $3 \mathrm{D}$ distribution of the Nafion layer on the $\mathrm{CB}$ is highlighted in the surface-rendered tomograms for samples 1 (Fig. 2c) and 2 (Fig. 2d): the $\mathrm{Cs}^{+}$ionomer is represented in blue and the $\mathrm{CB}$ support in transparent grey (see Supplementary Movie 1 and Supplementary Movie 2 for 3D view of sample 1 and sample 2, respectively). Qualitatively, a nonuniform distribution of the ionomer layer is revealed for both samples and a larger fraction of area of the CB appears to be coated by the ionomer in sample 1 as compared with sample 2 . To gain further insight into the differences in the coated area between the two samples, a quantification of the binarized tomograms was performed.

Ionomer layer morphological feature from electron tomogram. Important parameters that can be extracted from the segmented volume are the volume fraction, the degree of coverage and the average thickness of the ionomer layer (Table 1). The measured volume fraction of Nafion in sample 1 is around two times larger than that for sample 2 as expected from the ink composition. However, the total ionomer volume fraction measured on the two samples is smaller than the expected one: 20 and $13 \%$ instead of 33 and $17 \%$ for samples 1 and 2, respectively. One possible 

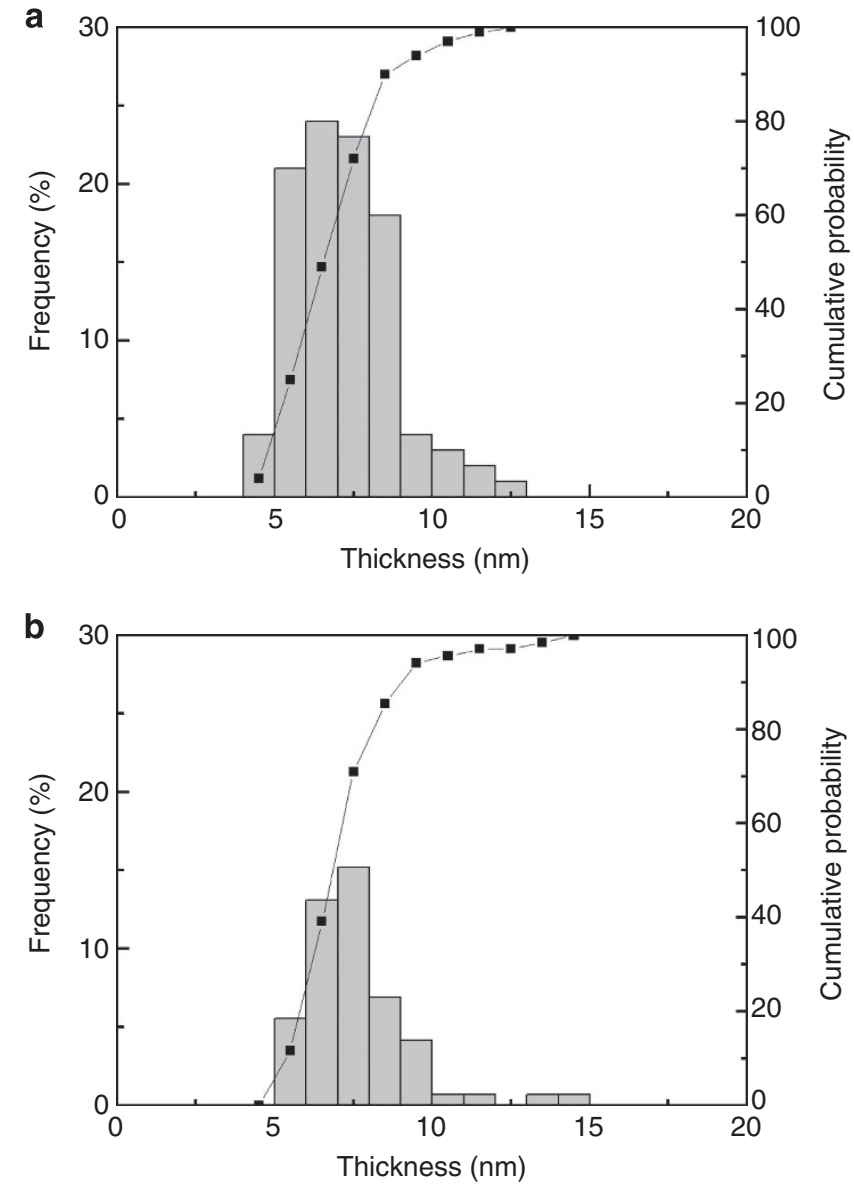

Figure 3 | Thickness of the Nafion layer. (a) Distribution of the thickness of the Nafion layer deposited on $C B$ for sample 1 (Nafion/CB $=0.5 \mathrm{w} / \mathrm{w}$ ). The histogram is derived from $\sim 100$ measurements performed in different zones of the reconstructed tomogram. The black dotted curve corresponds to the cumulative distribution of the ionomer layer thickness. (b) Same for sample 2 (Nafion/CB $=0.2 \mathrm{w} / \mathrm{w})$.

explanation to this discrepancy is that a significant fraction of the Nafion present in the ink agglomerates to form ionomer 'pockets' in the electrodes, as was observed by More et al. ${ }^{12}$ The formation of these Nafion pockets might be a direct consequence of the broad distribution of ionomer particle sizes ranging up to micrometre large aggregates in the Nafion dispersions ${ }^{24}$. Thus, our present results indicate that for sample 1 only $\sim 60 \%$ (and $76 \%$ for sample 2) of the ionomer present in the ink is available for covering uniformly the $\mathrm{CB}$ particles.

Other important parameters as the average thickness of the ionomer and the degree of coverage $(\delta)$ of the CB nanoparticles have also been quantified (Table 1). These parameters are known to have a high impact on the performance of the fuel cells as they play a crucial role in the transport processes of protons and gaseous reactants.

The thickness of the ionomer layer was quantified by a statistical analysis of the particle edges in the tomograms (Supplementary Fig. 4). The histograms, displayed in Fig. 3, are very close for the two samples: the average thickness amounts to 7.2 and $7.6 \mathrm{~nm}$ for samples 1 and 2, respectively, with $\sim 95 \%$ of the measured thicknesses being within the range of $4.5-9.5 \mathrm{~nm}$ in both cases. Such values are not only in good agreement with those previously reported in the literature ${ }^{12,13}$ but are also consistent with the ability of Nafion to form thin films and to coat evenly any media whether hydrophilic or hydrophobic ${ }^{25-28}$. Such a

\begin{tabular}{|c|c|c|c|}
\hline Sample & $\begin{array}{c}\text { Volume } \\
\text { fraction (\%) }\end{array}$ & $\begin{array}{c}\text { Surface } \\
\text { coverage }(\%)\end{array}$ & $\begin{array}{l}\text { Average layer } \\
\text { thickness (nm) }\end{array}$ \\
\hline \multicolumn{4}{|l|}{ Sample 1} \\
\hline Nafion $/ C B=0.5 \mathrm{w} / \mathrm{w}$ & $20.3 \pm 2.0$ & $79.5 \pm 2.3$ & $7.2 \pm 2.1$ \\
\hline \multicolumn{4}{|l|}{ Sample 2} \\
\hline Nafion $/ C B=0.2 \mathrm{w} / \mathrm{w}$ & $13.6 \pm 0.2$ & $49.9 \pm 1.2$ & $7.6 \pm 2.2$ \\
\hline \multicolumn{4}{|c|}{$\begin{array}{l}\text { CB, carbon black; HAADF-STEM, high-angle annular dark-field scanning transmission electron } \\
\text { microscope. } \\
\text { The error margins derive from using different smoothing procedures in the binarization process } \\
\text { of the 3D-reconstructed volumes, see Supplementary Fig. } 2 \text {. The CB surface coverage } \delta \text { is } \\
\text { calculated with the following formula: } \delta(\%)=\text { (area of the ionomer layer/surface of CB } \\
\text { nanoparticles) } \times 100 \text {. }\end{array}$} \\
\hline
\end{tabular}

property has been recently confirmed through the fabrication and characterization of physical-chemical properties of Nafion films with a thickness of several nanometres ${ }^{29,30}$. Moreover, the thickness of the layer derived from the present study corresponds to the smallest characteristic dimension of polymer aggregates in Nafion dispersions ${ }^{31}$. In suspensions, Nafion forms elongated aggregates similar to that of ribbons with a rectangular cross-section of $\sim 3$ by $10 \mathrm{~nm}$ as was evidenced by small angle $\mathrm{X}$-ray and neutron scattering ${ }^{31}$. This means that the shape of these aggregates does not change significantly upon processing the ink and they are deposited homogeneously on the $\mathrm{CB}$ particles until their entire surface is covered. In the present work, the ionomer distribution analysis was purposely performed using an ink consisting of $\mathrm{CB}$ without $\mathrm{Pt}$ nanoparticles as these would give a too high contrast in the tomograms as compared with the Nafion layer and the CB particles. We thus performed highresolution TEM additional analysis on a scratched real catalyst layer (Supplementary Fig. 5). Although the Nafion layer is not always easily observed in high-resolution TEM mode, the presence of $\mathrm{Pt}$ nanoparticles does not appear to affect the coverage of the $\mathrm{CB}$ nanoparticles by the Nafion layer; on these images, the Nafion layer thickness is in the same range as the one measured when performing tomography on the model system (Fig. 2).

On the other hand, the tomography analysis clearly shows a large difference between the two samples in terms of the covered area $\delta$ (in \%) of the supporting $\mathrm{CB}$ nanoparticles: whereas it amounts to $79.5 \pm 2.3 \%$ for sample 1 , it is only $49.9 \pm 1.2 \%$ for sample 2 (Table 1). Therefore, dividing the amount of ionomer by a factor of two in the processing ink does not influence the thickness of the layer of ionomer but rather leads to a lower coverage of the $\mathrm{CB}$ nanoparticles.

\section{Discussion}

These quantitative data provided by electron tomography on the Nafion thin layer can be directly related to the variations of the electrochemical performance of the related electrode and of the derived fuel cell with the amount of ionomer in the processing ink. It is indeed reported that the optimal Nafion content in the catalytic ink is $30 \pm 6 \% \mathrm{wt} \%{ }^{32}$. Lee et al. ${ }^{10}$, through measurements of the electrochemically active surface area (number of $\mathrm{Pt}$ nanoparticles in contact with the ionomer electrolyte), showed that the connectivity of the proton conduction paths is improved when the Nafion content increases from 23 to $40 \%$. They explained their results by a larger number of Nafion-coated Pt nanoparticles, while the polymer film thickness remains constant. This is in agreement with our present $3 \mathrm{D}$ analysis. We therefore propose the 
following scenario: (i) for a low Nafion content, the $\mathrm{CB}$ grains are partially covered by Nafion; thus, only a reduced percentage of $\mathrm{Pt}$ nanoparticles will be in contact with the ionomer, providing poor connectivity and therefore inducing a higher resistance to the proton flow into the active layer towards the membrane. (ii) For the optimal Nafion content, nearly all CBs are covered by Nafion, thus leading to a larger electrochemically active area. The thickness layer remains constant, around $7 \mathrm{~nm}$, and does not restrain $\mathrm{O}_{2}$ diffusion. Eventually, (iii) a further increase in the Nafion content leads to a thicker ionomer layer, which then becomes a significant $\mathrm{O}_{2}$ diffusion barrier leading to deteriorated performances.

To conclude, HAADF-STEM electron tomography was successfully used to image Nafion ultrathin layers in the electrodes of PEMFCs. This is the first experimental study of the Nafion layer in fuel cell electrodes providing quantitative morphological parameters (average thickness, degree of coverage of the CB-supporting particles). These results allow a direct correlation of the 3D Nafion layer structure to the electrochemical response of the electrode. This accurate 3D analysis is of great interest for describing the electrode microstructure, such description being needed for electrochemical models aiming at predicting the performance of a fuel cell. An extension of the present work will be carried out to study the impact on the ionomer layer structure, and hence on the electrode properties, using different types of ionomer as well as other ink or electrodemanufacturing processes. In addition, the present methodology based on nanoscale tomography analysis should be of interest for many other problems where the surface coverage and the $3 \mathrm{D}$ structure of nano-objects and nanomaterials by a thin nanometre-thick layer is involved such as in nanocomposite material batteries, third-generation organic and hybrid solar cells or new catalysts for environmental catalysis.

\section{Methods}

Sample preparation. Slurries have been prepared by mixing CB nanoparticles Vulcan XC72 from Cabot with commercial suspension of Nafion D2020 from Dupont $(20 \mathrm{wt} \%$ in water and isopropanol mixture of ionomer with an equivalent weight of $1,000 \mathrm{~g} \mathrm{eq}^{-1}$ ) into a mixture of pure ethanol and ultrapure water. The dry content was $23 \mathrm{wt} \%$. Samples have been fabricated by coating the slurries on a commercial Gas Diffusion Layer SGL24BC from SGL Carbon. Two samples have been prepared with 17 and $33 \mathrm{wt} \%$ of Nafion in the ink.

To perform the tomographical HAADF-STEM analysis of the Nafion deposited on $\mathrm{CB}$ nanoparticles, a small part of the GDL coating was scratched and dispersed in a saturated solution of $\mathrm{Cs}_{2} \mathrm{SO}_{4}$ in water during $72 \mathrm{~h}$ in order to exchange the sulfonic acid sites by $\mathrm{Cs}^{+}$. We used $\mathrm{Cs}^{+}$cations rather than uranyl $\mathrm{UO}_{2}^{2+}$ ones because their monovalent character allows a double loading as compared with divalent cations. Floating particles of $\mathrm{CB}$ were then transferred on lacey carboncoated Cu TEM grids (Agar Ref: S166-3).

Electron tomography. HAADF-STEM electron tomography was carried out on a LB FEI TITAN 80-300 electron microscope equipped with a probe $\mathrm{C}_{\mathrm{s}}$ corrector operating at $200 \mathrm{kV}$ and using a Fischione 2020 single-tilt tomography holder. Before recording a series of images, aberrations of the condenser lenses were corrected up to second order using the Zemlin tableau. A convergence angle of $10 \mathrm{mrad}$ and $128 \mathrm{~mm}$ of camera length were used. Using the '3D Explorer' FEI software, the data collections were performed by tilting the specimen around a single axis perpendicular to the electron beam between $-76^{\circ}$ and $+72^{\circ}$ for $\mathrm{Cs}^{+}$. ionomer- 1 , and between $-72^{\circ}$ and $+66^{\circ}$ for $\mathrm{Cs}^{+}$-ionomer- 2 and using $2^{\circ}$ steps. The range of achievable tilting angles is limited by the shadowing effect of the grid when using too large angles. In order to reconstruct tomograms, the registered tilt series were aligned using the FEI Inspect $3 \mathrm{D}$ software and a software written in MATLAB. In particular, compressed sensing algorithm was applied as described in the Supplementary Information. The reconstructed volumes were thereafter processed using the Avizo 6.0 Fire edition and the Fiji software, further details being provided in the Supplementary Information.

\section{References}

1. Kerr, R. A. Do we have the energy for the next transition? Science 329, 780-781 (2010).

2. Tour, J. M., Kittrell, C. \& Colvin, V. L. Green carbon as a bridge to renewable energy. Nat. Mater. 9, 871-874 (2010).
3. Steele, B. C. H. \& Heinzel, A. Materials for fuel-cell technologies. Nature 414, 345-352 (2001).

4. Debe, M. K. Electrocatalyst approaches and challenges for automotive fuel cells. Nature 486, 43-51 (2012).

5. Litster, S. \& McLean, G. PEM fuel cell electrodes. J. Power Sources 130, 61-76 (2004).

6. Greeley, J. et al. Alloys of platinum and early transition metals as oxygen reduction electrocatalysts. Nat. Chem. 1, 552-556 (2009).

7. Diat, O. \& Gebel, G. Proton channels. Nat. Mater. 7, 13-14 (2008)

8. Kumar, A., Ciucci, F., Morozovska, A. N., Kalinin, S. V. \& Jesse, S. Measuring oxygen reduction/evolution reactions on the nanoscale. Nat. Chem. 3, 707-713 (2011).

9. Li, J., Park, J. K., Moore, R. B. \& Madsen, L. A. Linear coupling of alignment with transport in a polymer electrolyte membrane. Nat. Mater. 10, 507-511 (2011).

10. Lee, M. et al. New evaluation method for the effectiveness of platinum/carbon electrocatalysts under operating conditions. Electrochim. Acta 55, 8504-8512 (2010).

11. Berejnov, V., Susac, D., Stumper, J. \& Hitchcock, A. P. 3D Chemical mapping of PEM fuel cell cathodes by scanning transmission soft $\mathrm{x}$-ray spectrotomography. ECS Trans. 50, 361-368 (2012).

12. More, K. L., Borup, R. \& Reeves, K. S. Identifying contributing degradation phenomena in PEM fuel cell membrane electrode assemblies via electron microscopy. ECS Trans. 3, 717-733 (2006).

13. More, K. L. \& Reeves, K. S. TEM specimen preparation of partially-embedded electrodes from proton exchange membrane fuel cell membrane electrode assemblies. Microsc. Microanal. 11(Suppl. 2): 2104-2105 (2005).

14. Midgley, P. A. \& Dunin-Borkowski, R. E. Electron tomography and holography in materials science. Nat. Mater. 8, 271-280 (2009).

15. Pennycook, S. J. \& Boatner, L. A. Chemically sensitive structure-imaging with a scanning-transmission electron microscope. Nature 336, 565-567 (1988).

16. Cirkel, P. A., Okada, T. \& Kinugasa, S. Equilibrium aggregation in perfluorinated ionomer solutions. Macromolecules 32, 531-533 (1999).

17. Oosterhout, S. D. et al. The effect of three-dimensional morphology on the efficiency of hybrid polymer solar cells. Nat. Mater. 8, 818-824 (2009).

18. Schulenburg, H. et al. 3D imaging of catalyst support corrosion in polymer electrolyte fuel cells. J. Phys. Chem. C 115, 14236-14243 (2011).

19. Grothausmann, R. et al. Quantitative structural assessment of heterogeneous catalysts by electron tomography. J. Am. Chem. Soc. 133, 18161-18171 (2011)

20. Banham, D. et al. Effect of Pt-loaded carbon support nanostructure on oxygen reduction catalysis. J. Power Sources 196, 5438-5445 (2011).

21. Gontard, L. C., Dunin-Borkowski, R. E. \& Ozkaya, D. Three-dimensional shapes and spatial distributions of $\mathrm{Pt}$ and $\mathrm{PtCr}$ catalyst nanoparticles on carbon black. J. Microsc. 232, 248-259 (2008).

22. Scheiba, F., Benker, N., Kunz, U., Roth, C. \& Fuess, H. Electron microscopy techniques for the analysis of the polymer electrolyte distribution in proton exchange membrane fuel cells. J Power Sources 177, 273-280 (2008).

23. Goris, B., Roelandts, T., Batenburg, K. J., Mezerji, H. H. \& Bals, S. Advanced reconstruction algorithms for electron tomography: From comparison to combination. Ultramicroscopy 127, 40-47 (2013).

24. Friedrich, H., de Jongh, P. E., Verkleij, A. J. \& de Jong, K. P. Electron tomography for heterogeneous catalysts and related nanostructured materials. Chem. Rev. 109, 1613-1629 (2009).

25. Martin, C. W., Baker, S. H., Gordon, T. D. \& Davila, M. Processes for forming thin, durable coatings of cation-containing polymers on selected substrates. US Patent 5.718,947 (1998).

26. Martin, C. R., Rhoades, T. A. \& Ferguson, J. A. Dissolution of perfluorinated ion containing polymers. Anal. Chem. 54, 1639-1641 (1982).

27. Szentirmay, M. N., Campbell, L. F. \& Martin, C. R. Silane coupling for attaching Nafion to glass and silica. Anal. Chem. 58, 661-662 (1986).

28. Moore, R. B. \& Martin, C. R. Procedure for preparing solution-cast perfluorosulfonate ionomer films and membranes. Anal. Chem. 58, 2569-2570 (1986).

29. Paul, D. K., Fraser, A., Pearce, J. \& Karan, K. Understanding the ionomer structure and the proton conduction mechanism in PEFC catalyst layer: adsorbed nafion on model substrate. ECS Trans. 41, 1393-1406 (2011).

30. Paul, D. K., Fraser, A. \& Karan, K. Towards the understanding of proton conduction mechanism in PEMFC catalyst layer: conductivity of adsorbed Nafion films. Electrochem. Commun. 13, 774-777 (2011).

31. Loppinet, B., Gebel, G. \& Williams, C. E. Small-angle scattering study of perfluorosulfonated ionomer solutions. J. Phys. Chem. B 101, 1884-1892 (1997).

32. Xie, J. et al. Influence of ionomer content on the structure and performance of PEFC membrane electrode assemblies. Electrochim. Acta 55, 7404-7412 (2010).

\section{Acknowledgements}

We acknowledge financial support from the CEA NANOSCIENCE Program (Project TOMOENER). M.L.-H. thanks the MECD Spanish Ministry (ref. EX2010-1135) and the Eurotalents program funded by CEA and the Marie Curie Actions of the European 
Commission for funding his stay at CEA-Grenoble. We gratefully acknowledge Dr JeanChristophe Gabriel for fruitful and stimulating discussions.

\section{Author contributions}

M.L.-H. prepared the TEM samples, conducted the electron tomography experiments and the data analysis under the guidance of L.G. and F.C. T.P. participated in the tomogram reconstruction. A.M. and S.E. prepared the fuel cell electrodes and assisted with the interpretation of the data. G.G. helped in the experiment definition and the data interpretation. P.B.-G. and P.-H.J. participated in the experiments and their interpretation. All authors collaborated on the manuscript.

\section{Additional information}

Supplementary Information accompanies this paper at http://www.nature.com/ naturecommunications

Competing financial interests: The authors declare no competing financial interests.

Reprints and permission information is available online at http://npg.nature.com/ reprintsandpermissions/

How to cite this article: Lopez-Haro, M. et al. Three-dimensional analysis of Nafion layers in fuel cell electrodes. Nat. Commun. 5:5229 doi: 10.1038/ncomms6229 (2014). 